\title{
Site-specific adsorption of gold nanoparticles coated with thermo-responsive peptides
}

\author{
Nobuyuki Higashi, Takayuki Ochiai, Chie Kanazawa and Tomoyuki Koga
}

A thermo-responsive elastin, elastin-like peptide (ELP)-gold nanoparticle (AuNP) hybrid (ELP-AuNP), was successfully prepared via the in situ reduction of $\mathrm{HAuCl}_{4}$ in the presence of lipoic acid-terminated ELP: (VPGVG) 4 (V:valine, P:proline, G:glycine) synthesized using a solid-phase peptide synthesis method. The conformation and aggregation properties of the ELP-AuNP in an aqueous buffer solution were examined using circular dichroism (CD) and ultraviolet-visible (UV-Vis) spectroscopies and transmission electron microscopy (TEM) observation. The phase transition of the ELP layer at the periphery of the AuNP because of the conformational change from random coil to $\beta$-turn structures was found to proceed gradually with increasing temperature and to simultaneously cause an aggregation of the ELP-AuNP because of the component's hydrophobic interactions and a color change of the solutions from red to violet. The ELP-AuNPs were aligned selectively onto patterned, hydrophobic polystyrene stripes on a glass plate when the plate was immersed and incubated in the solution containing the ELP-AuNP above its phase transition temperature, as verified using atomic force microscopy (AFM) and scanning electron microscopy (SEM).

Polymer Journal (2013) 45, 523-528; doi:10.1038/pj.2012.220; published online 12 December 2012

Keywords: $\beta$-turn conformation; elastin-like peptide; gold nanoparticle; phase transition; site-specific adsorption; thermo-responsiveness

\section{INTRODUCTION}

Elastin is one of the most important classes of naturally occurring structural proteins ${ }^{1-3}$ and is commonly found in the vascular walls, skin and lung tissue of mammals where it functions as an elastomeric materials. There are many types of elastin and tropoelastin, with VPGVG (V:valine, P:proline, G:glycine) as its prominent amino-acid repeat, is one of the most widely studied. $^{4,5}$ The aggregation, conformational and mechanical properties of both chemically synthesized ${ }^{6,7}$ and recombinantly prepared poly(VPGVG) ${ }^{8-12}$ (an ELP, elastin-like polypeptide) have been extensively investigated. ELPs exhibit a reversible hydrophilic -hydrophobic phase transition in aqueous solution in response to temperature changes: ELPs are water soluble at room temperature, but as the temperature increases, their solubility decreases. This remarkable lower critical solution temperature behavior is a result of the conformational change in the ELP molecule from a random coil to a $\beta$-spiral including a type type-II $\beta$-turn conformation, which is caused by the hydrophobic dehydration of the valine side chains. ${ }^{13,14}$ This inverse phase transition of the ELPs is analogous to the lower critical solution temperature transition exhibited by poly $(N$-isopropylacrylamide $)$ and its copolymers in aqueous solutions. ${ }^{15-17}$ The ELPs potentially offer several advantages over poly $(\mathrm{N}$-isopropylacrylamide) such as biocompatibility and biodegradablility characters. The ELPs enable important macromolecular properties such as the sequence, chain length and stereochemistry to be controlled to an extent that is impossible with synthetic polymers. Furthermore, the type, number and location of amino-acid residues in the polypeptide chain can be precisely specified, which allows not only an expansion of the transition temperature range but also facilitated functionalization.

We report the coating of gold nanoparticles (AuNPs) with these thermo-responsive ELPs and the control of their aggregation state based on the ELP coatings. Nanoparticles show exhibit interesting optical and electronic properties as a consequence based on their dimensions. The dimensions of these particles make them ideal candidates for the fabrication of functional nanostructures. Among metallic nanoparticles, AuNPs have an easily modified surface and are detectable spectroscopically because of their surface plasmon resonance. AuNPs provide also prominent biocompatibility even for clinical use because of their low cytotoxicity. We previously reported that the successful preparation of well-dispersed helical poly $(\mathrm{L}-$ glutamic acid)-coated AuNPs and their immobilization without aggregation in the $\beta$-sheet peptide network with poly(L-lysine) using a layer-by-layer deposition method. ${ }^{18}$

In this study, we present an effective and facile approach for preparing self-assembled AuNP arrays guided with ELP coatings, 
which are expected to thermally switch between hydrophilic and hydrophobic phases. An alternative technique that patterns the metal nanoparticles via directed self-assembly using appropriate templates was required because the conventional top-down methodologies, such as optical lithography, are limited in their resolution. Sita et al. ${ }^{19}$ have found out that phase-separated copolymers used as templates can direct the self-assembly of alkane thiol-coated AnNPs. We are similarly interested in the patterning of AuNPs via directed selfassembly. A pentad repeat (VPGVG) $)_{n}(n=4)$ that provides sufficient chain length to cause a conformational change in response to a thermal stimulus ${ }^{20}$ was chosen as the ELP segment and synthesized via conventional solid-phase synthesis. At the $N$-terminal of the $(\mathrm{VPGVG})_{4}$, lipoic acid was attached to the gold surface through a gold -thiolate bond (Figure 1). The thermo-responsive behavior of

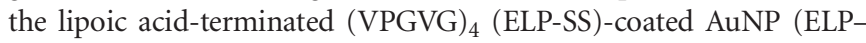
AuNP) was investigated using circular dichroism (CD) and surface plasmon spectroscopies. The selective adsorption of these ELP-AuNPs was examined using micro-patterned hydrophobic polystyrene (PS) on a glass plate that was prepared using the micro-moldering in capillaries method. ${ }^{21}$

\section{EXPERIMANTAL PROCEDURE}

\section{General procedures}

The ${ }^{1} \mathrm{H}$ NMR spectra were measured on a $400-\mathrm{MHz}$ JEOL FT-NMR AL400 instrument (JEOL, Tokyo, Japan). The matrix-assisted laser desorption/ ionization time-of-flight mass spectrometry spectra were measured on a Shimadzu AXIMA-CFR plus mass spectrometer (Shimadzu, Kyoto, Japan) using 2,5-dihydroxybenzoic acid as the matrix. The $\mathrm{CD}$ spectra were measured on a Jasco J-820 spectropolarimeter (JASCO, Tokyo, Japan) with a temperature control unit. The concentrations of the peptide solutions were determined using absorbance at approximately $215 \mathrm{~nm}$ due to the band of the peptide bond. The UV-Vis spectra were measured using a Shimadzu UV-2100 spectrophotometer (Shimadzu) with a temperature control unit. The water contact angle for the surface characterization of the samples was measured using a FACE CA-D contact angle meter (Kyowa Interface Science, Saitama, Japan) at ambient temperature. Ultra-pure water was added dropwise onto the samples. The average of five measurements taken at different positions on each sample was adopted.

\section{Materials}

Fmoc (9-fluorenylmethoxycarbonyl) amino acids, $\mathrm{HAuCl}_{4}$ and other chemicals were used as received.

Lipoic acid-terminated (VPGVG) ${ }_{4}$ (ELP-SS) was prepared using a standard solid-phase synthesis method using with Fmoc-chemistry. The peptide chain was synthesized on an Alko Resin with Fmoc-amino-acid derivatives (3 equiv), D, L-lipoic acid (3 equiv) and 1,3-diisopropylcarbodiimide (3 equiv) in dimethylformamide for coupling, and piperidine (25\%)/dimethylformamide for Fmoc group removal. To cleave the lipoic acid-functionalized peptide from the resin, the peptide resin was treated with trifluoroacetic acid/ $\mathrm{CH}_{2} \mathrm{Cl}_{2}(9: 1 \mathrm{v} / \mathrm{v})$. The asobtained crude peptide thus obtained was purified by via high-performance liquid chromatography. The purified peptide was identified by using matrixassisted laser desorption/ionization time-of-flight mass spectrometry and ${ }^{1} \mathrm{H}$ NMR spectroscopies. ${ }^{1} \mathrm{H}$ NMR (DMSO- $d_{6}$, tetramethylsilane): $\delta$ 0.8-1.0 $\left(\gamma-\mathrm{CH}_{3}\right.$ Val); 1.3-2.2 $\left(\mathrm{CO}\left(\mathrm{CH}_{2}\right)_{4} \mathrm{CH}, \mathrm{CHCH}_{2} \mathrm{CH}_{2}, \beta, \gamma-\mathrm{CH}_{2}\right.$ Pro and $\beta-\mathrm{CH}$ Val $) ; 3.5-3.8$ ( $\delta$ - $\mathrm{CH}_{2}$ Pro and $\mathrm{CH}_{2}$ Gly); 4.1-4.8 ( $\alpha-\mathrm{CH}$ Val and $\alpha-\mathrm{CH}$ Pro); 7.4-8.4 (NH Val and Gly). Matrix-assisted laser desorption/ionization time-of-flight mass spectrometry: $m / z 1843.7[\mathrm{M}+\mathrm{H}]^{+} / 1845.3[\mathrm{M}+\mathrm{H}]{ }^{+}$calcd.

The ELP-SS-coated AuNP (ELP-AuNP) was simply prepared using a conventional technique ${ }^{22}$ with sodium borohydride $\left(\mathrm{NaBH}_{4}\right)$ as the reducing agent. A $1 \%$ aqueous $\mathrm{NaBH}_{4}$ solution $(10 \mathrm{ml})$ was added to a $0.2 \%$ aqueous $\mathrm{HAuCL}_{4}$ under ice cooling. The mixture was stirred at a constant rate of 1500 r.p.m., at $0{ }^{\circ} \mathrm{C}$ for $3 \mathrm{~h}$. The reaction mixture was then centrifuged three times at 15000 r.p.m., and $5^{\circ} \mathrm{C}$ for $30 \mathrm{~min}$, to remove the free ELP-SS. The characterization of the as-obtained ELP-AuNP was performed via CD, UV-Vis spectroscopies and TEM.

The patterning of PS on a glass plate was carried out according to the micromoldering in capillaries method. ${ }^{21}$ The elastomeric stamps were first fabricated by replica molding of polydimethylsiloxane on a commercially available compact disk (CD-R). The as-obtained polydimethylsiloxane stamp was kept on glass plate, and an aliquot of PS solution (1\% in toluene) was dropped at one edge of the stamp to spontaneously fill the channels by capillary action. The apparatus was then heated at $130{ }^{\circ} \mathrm{C}$ (above the $T_{\mathrm{g}}$ (glass transition temperature) of PS) for $30 \mathrm{~min}$. After cooling to room temperature, the stamp was removed.

\section{Microscopies}

The TEM images were obtained using a JEOL JEM-2100F transmission electron microscope operating with an accelerating voltage of $200 \mathrm{kV}$. All TEM samples were prepared by drop casting the diluted ELP-AuNP solution onto a $\mathrm{Cu}$ TEM grid. The grid was then dried overnight at ambient temperature. The SEM images were obtained using a JEOL JSM-7000FD instrument (JEOL) operating with an accelerating voltage of $15 \mathrm{kV}$. The slightly pressed specimens were sputter coated with gold. AFM was performed in the tapping mode using a Nanoscope IIIa (Digital Instruments, Santa Barbara, CA, USA) with silicon tips at a line frequency of $1 \mathrm{~Hz}$.

\section{RESULTS AND DISCUSSION}

\section{Characterization of ELP-AuNP}

The peptide-AuNP hybrid (ELP-AuNP) was successfully prepared by the reduction of $\mathrm{HAuCl}_{4}$ with $\mathrm{NaBH}_{4}$ in the presence of ELP-SS. The ELP-AuNP was dissolved in water at an acidic $\mathrm{pH}$ so that the terminal $\mathrm{COOH}$ group of the ELP-SS was protonated to provide a stable colloidal solution. The size of the gold colloids, as determined via TEM, was $8 \pm 2 \mathrm{~nm}$ (Figure 2a). The UV-Vis spectrum of the colloidal solution exhibited a surface plasmon absorption peak at $530 \mathrm{~nm}$, as shown in Figure $2 \mathrm{~b}$.

The temperature dependences of the ELP-AuNP secondary structure in dilute solution $(50 \mu \mathrm{M})$ were evaluated using CD spectroscopy. For comparison, the same experiment was performed on AuNP-free ELP-SS, and the spectra obtained in a phosphate buffer ( $\mathrm{pH} 3.3)$ at

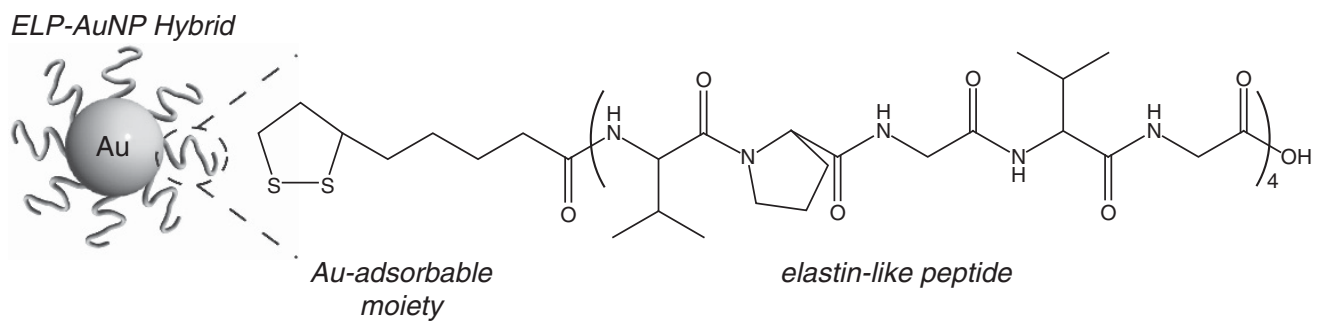

Figure 1 Structural model for the hybrid of an elastin-like peptide and a gold nanoparticle (ELP-AuNP). A full color version of this figure is available at Polymer Journal online. 
different temperatures are shown in Figure 3a. At low temperature $\left(10^{\circ} \mathrm{C}\right)$, the spectrum yielded a pattern typical of a random coil conformation with a negative maximum at approximately $196 \mathrm{~nm}$. The molar ellipticity [0] value at $196 \mathrm{~nm}$ decreased with increasing temperature, together with a smaller decrease in the $[\boldsymbol{\theta}]$ value between 206 and $212 \mathrm{~nm}$ and an increase at $222 \mathrm{~nm}$. A positive peak in the region between 206 and $212 \mathrm{~nm}$ is characteristic of a type-II $\beta$-turn conformation. A similar temperature-dependent spectral change was observed for the aqueous solution of ELP-AuNP. Figure $3 \mathrm{~b}$ displays the conformational transition curves for ELP-AuNP and AuNP-free ELP-SS under the same conditions. For both ELPs, the conformational transition from random coil to $\beta$-turn occurred gradually over a wide temperature range from 5 to $60^{\circ} \mathrm{C}$, and the two ELPs provided similar temperature profiles with almost no difference in the $[\boldsymbol{\theta}]$ value. Thus, one can concluded that the thermo-responsiveness of the
ELP conformation is not significantly affected by anchoring to the AuNP surface.

\section{Thermo-responsive assembly of ELP-AuNP}

The phase transition behavior of ELP-AuNP was monitored by measuring the absorption spectrum as the solution temperature increased from 10 to $70^{\circ} \mathrm{C}$ (Figure 4a). The spectrum of ELPAuNP displayed an absorption maximum at $530 \mathrm{~nm}$ and $10^{\circ} \mathrm{C}$, and the spectrum did not change significantly as the solution temperature increased from 10 to $40^{\circ} \mathrm{C}$. Temperature increases above $40^{\circ} \mathrm{C}$, caused a red shift of the peak wavelength, along with broadening of the spectrum. We also measured the temperature-dependent absorption spectrum for the peptide (ELP)-free, bare AuNP as a control, which is commercially available as a citric acid-stabilized AuNP with a size of $10 \mathrm{~nm}$ that is consistent with that of our ELP-AuNP a

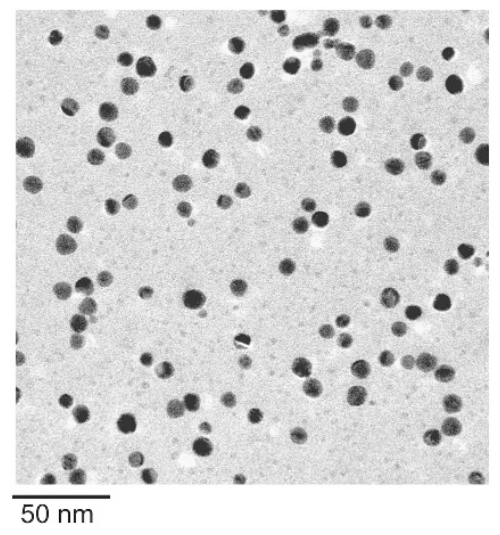

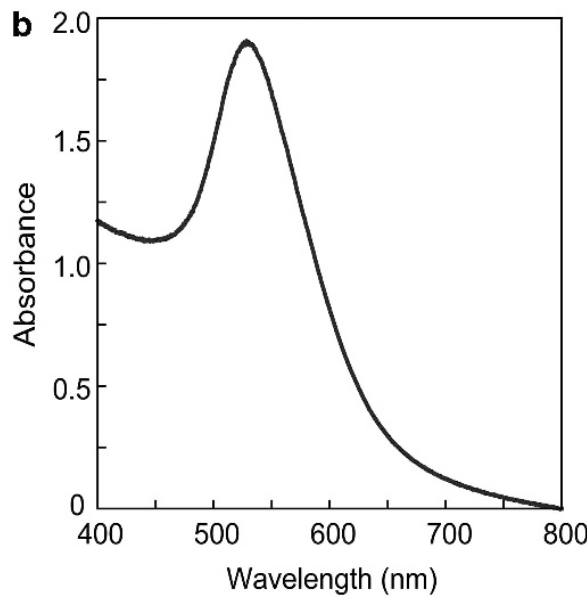

Figure 2 (a) Transmission electron microscopy image of an elastin-like peptide and a gold nanoparticle (ELP-AuNP). The sample was prepared by drop casting an ELP-AuNP solution onto a Cu grid at $10^{\circ} \mathrm{C}$. (b) The visible absorption spectrum of ELP-AuNP in a $5 \mathrm{~mm}$ phosphate buffer at pH 3.3 and $10^{\circ} \mathrm{C}$. $(E L P-A u N P)=0.1 \mathrm{mg} \mathrm{ml}^{-1}$.
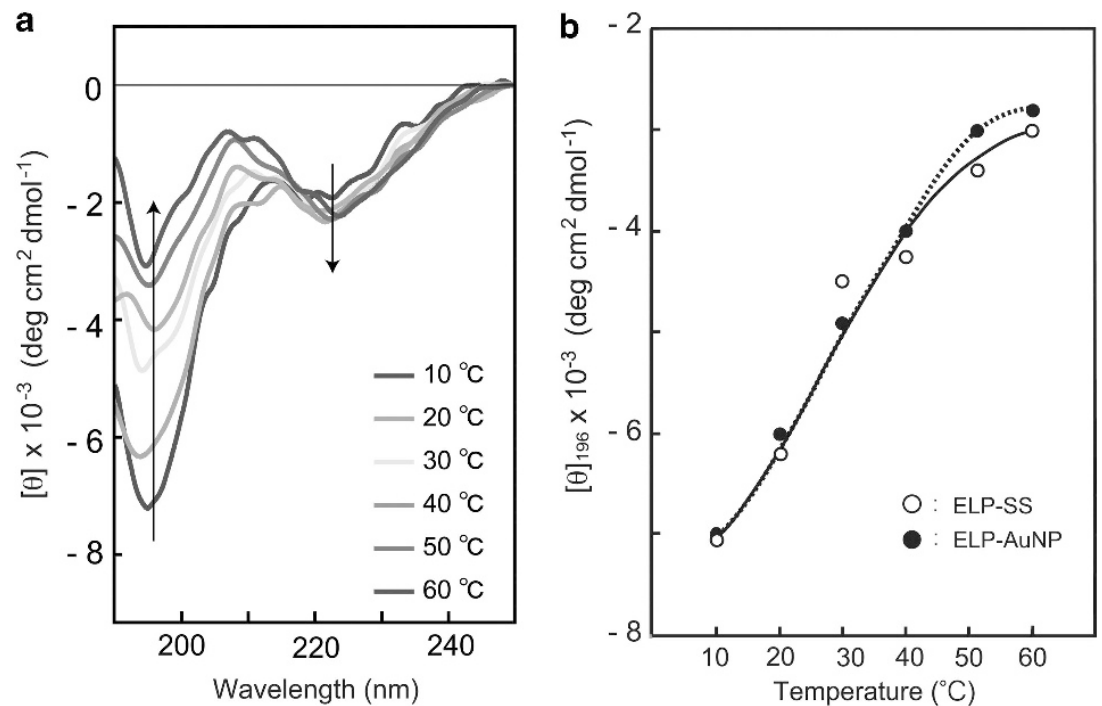

Figure 3 (a) Temperature dependence of the circular dichroism (CD) spectra of elastin-like peptide labeled with lipoic acid at N-terminal (ELP-SS) in a $5 \mathrm{~mm}$ phosphate buffer at $\mathrm{pH} 3.3[\mathrm{ELP}-\mathrm{SS}]=50 \mu \mathrm{m}$. (b) Temperature profile of $[\theta]_{196}$ values for ELP-SS $(O)$ and ELP-AuNP $(\mathbf{O})$ during heating. A full color version of this figure is available at Polymer Journal online. 

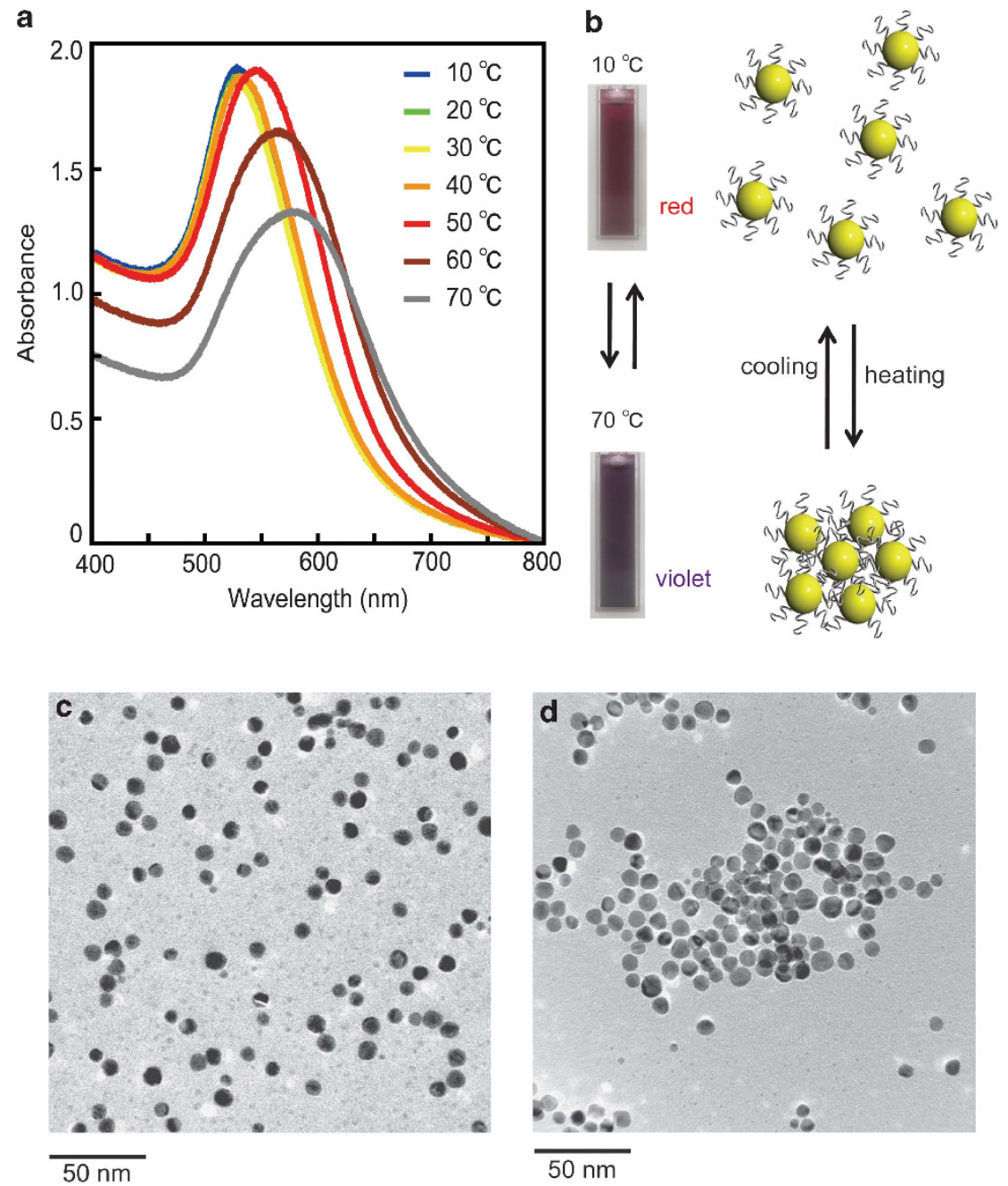

Figure 4 (a) Temperature-dependent absorption spectral changes for the elastin-like peptide and gold nanoparticles (ELP-AuNPs) in a 5 mm phosphate buffer at $\mathrm{pH} 3.3$ during heating. [ELP-AuNP] $=0.1 \mathrm{mg} \mathrm{ml}^{-1}$. (b) Schematic diagram of the reversible aggregation of gold nanoparticles caused by the thermo-triggered phase transition of the ELP shell: at $10^{\circ} \mathrm{C}$ the ELP-AuNPs in the hydrophilic phase exhibit the red color typical of isolated gold colloids, and at $70^{\circ} \mathrm{C}$ the aggregates exhibit a violet color. (c, d) Transmission electron microscopy images of the ELP-AuNPs prepared at $10^{\circ} \mathrm{C}(\mathbf{c})$ and $70^{\circ} \mathrm{C}$ (d). The image in (c) is the same as that cited in Figure 1a.

$(8 \pm 2 \mathrm{~nm})$. No change was observed in the absorption spectrum of the control as a function of temperature, which strongly suggested that the spectral changes observed for ELP-AuNP are due to the temperature-dependent phase transition of the ELP layer at the periphery of the AuNP. These drastic spectral changes as a function of temperature can be attributed to the long-range coupling of surface plasmons. Below the phase transition temperature, the ELP-AuNP colloids in solution have an average interparticle distance large enough to appear red in color because of the surface plasmon resonance of the individual colloidal particles. Raising the temperature of the ELP-AuNP solution, cause the ELP layer to undergo an intramolecular hydrophilic-hydrophobic transition, resulting in an enhanced hydrophobic interaction between the colloids and the subsequent formation of large aggregates. The interparticle distance in the aggregate state decreases, and a long-range coupling of plasmons is observed, which results in a red shift in the absorption spectrum, and the color of solution appears violet (Figure $4 \mathrm{~b}$ ). Similar spectral changes have been experimentally observed, ${ }^{23}$ and theoretically explained ${ }^{24}$ for the formation of colloidal aggregates of
AuNPs. The observed aggregation of ELP-AuNP may be caused by the dense assembly of ELP molecules onto the AuNP surface because a segment length as short as the 20-residue ELP does not exhibit coacervation in itself.

To obtain direct evidence of the ELP-AuNP aggregation, the aggregates were observed using TEM and the pictures, taken below and above the phase transition temperature, are shown in Figures $4 \mathrm{c}$ and d. At $70{ }^{\circ} \mathrm{C}$ above the phase transition temperature, most of the particles are clearly shown in the aggregate state. Although they are individually dispersed at $10{ }^{\circ} \mathrm{C}$, the existence of a few aggregates may not be completely excluded at this temperature. These TEM pictures strongly support the color change because of surface plasmon coupling caused by aggregation of ELP-AuNP that is presented in Figures $4 \mathrm{a}$ and $\mathrm{b}$.

\section{Specific adsorption of ELP-AuNP onto a patterned solid surface} Finally, we demonstrate the site-specific adsorption of ELP-AuNP using the thermo-switchable character of the ELP layer. To elucidate the selective adsorption of ELP-AuNP onto a solid substrate, we 
prepared two solid substrates, a hydrophobic glass plate treated with octadecyltriethoxysilane and an untreated bare glass plate, providing hydrophobic (water contact angle: $100^{\circ}$ ) and hydrophilic (water contact angle: $20^{\circ}$ ) surfaces, respectively. The interaction of these glass plates with ELP-AuNP was examined by separately immersing them into colloidal solutions containing ELP-AuNP overnight at $70{ }^{\circ} \mathrm{C}$ above the phase transition temperature and measuring the UVVis spectra for both plates. The absorption peaks due to the surface plasmon resonance of AuNP were observed for both glass plates, indicating the adsorption of ELP-AuNP onto both (data not shown). However, the peak absorbances were quite different: that for the hydrophobic glass plate was larger than that for the hydrophilic one by a factor of approximately 6 . The ELPs adopt a hydrophobic $\beta$-turn structure at $70^{\circ} \mathrm{C}$ and are more likely to interact with the hydrophobic glass plate than with the hydrophilic one. The maximum absorption for the hydrophobic plate was found to red shift up to $585 \mathrm{~nm}$ while that for the bare glass plate was located at $530 \mathrm{~nm}$, suggesting an aggregation of ELP-AuNPs. When the same experiment was performed at $10^{\circ} \mathrm{C}$ below the phase transition temperature, the changes in the absorbance for both plates were relatively small, and the difference in absorbance between the hydrophobic and hydrophilic plates was unremarkable compared with the results obtained at $70{ }^{\circ} \mathrm{C}$. Therefore, the thermo-triggered change between the hydrophilic and hydrophobic characteristics of the ELP layers is expected to have an important role in aligning ELP-AuNP on a hydrophobic, patterned surface via directed assembly. Figure 5a presents the SEM a

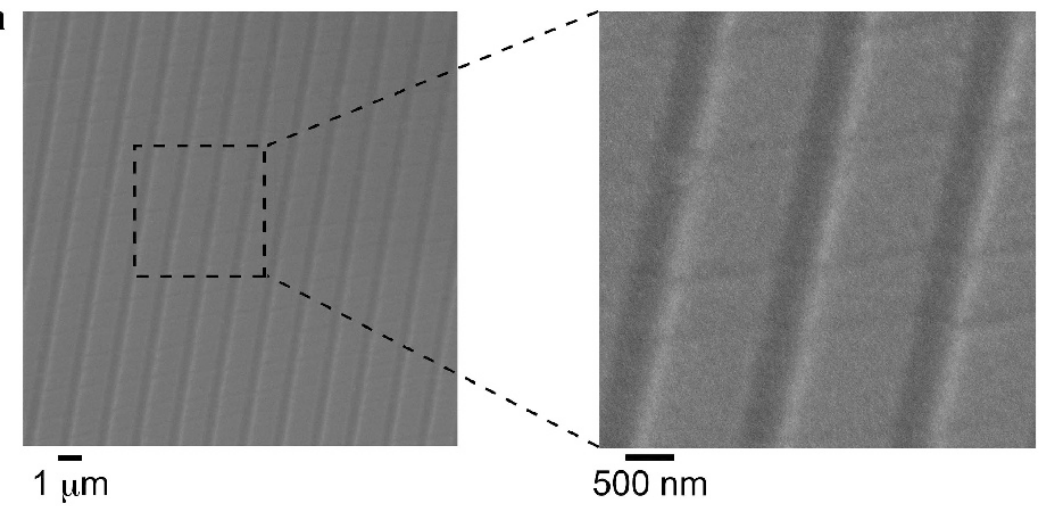

b
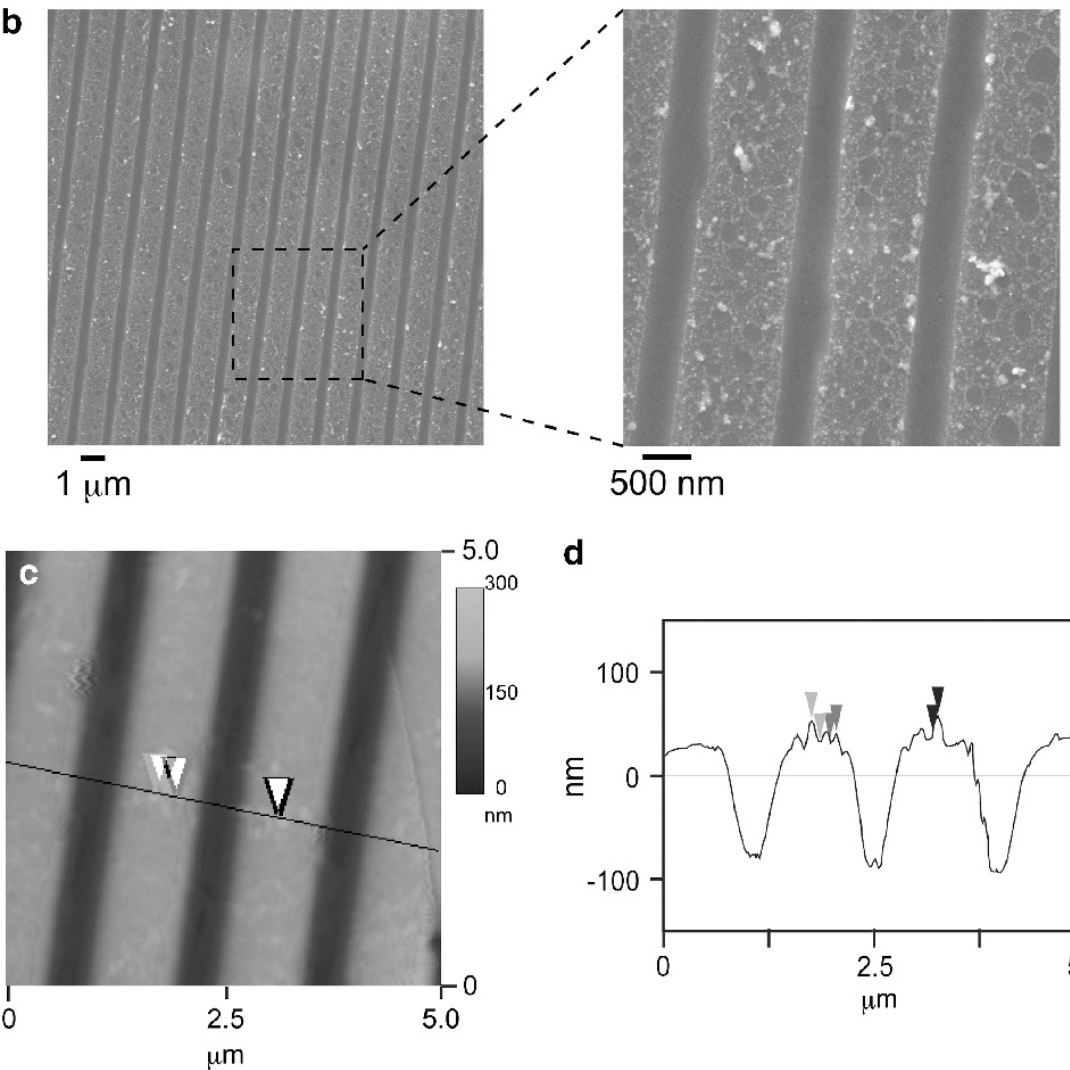

d

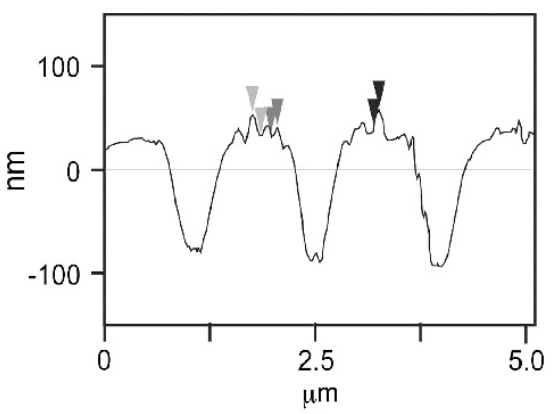

Figure 5 Scanning electron microscopy images of the patterned polystyrene (PS) surfaces (a) before and (b) after adsorption of the elastin-like peptide and a gold nanoparticle (ELP-AuNP). The PS patterning was performed on a glass plate using an micro-moldering in capillaries method. ${ }^{21}$ (c) Atomic force microscopy (AFM) image of the patterned PS surface after adsorption of the ELP-AuNPs. (d) AFM z-profile of the ELP-AuNP-adsorbed pattern. A full color version of this figure is available at Polymer Journal online. 
image for patterned PS on a glass plate prepared using a polydimethylsiloxane stamp derived from a compact disk as a master according to the micro-moldering in capillaries method. ${ }^{21}$ Sets of parallel lines can be seen in which the relatively bright regions correspond to the PS and the dark regions to the glass surface. This PS-patterned plate was immersed in the same aqueous solution of ELP-AuNPs as described above at $70^{\circ} \mathrm{C}$ overnight. After rinsing with pure water at $70{ }^{\circ} \mathrm{C}$ and drying, s.e.m. images of the sample were obtained and are shown in Figure 5b. In the s.e.m. image, we can observe additional bright adsorbates, which are gold particles and their aggregates, but only on the PS stripes. The topography of these adsorbates is made clear by the AFM image shown in Figure $5 \mathrm{c}$. In the image, bright adsorbates can again be seen on the PS ridge. The z-profile (Figure $5 \mathrm{~d}$ ) indicates that the cross-section of the adsorbates ranged from 6 to $17 \mathrm{~nm}$. These values are nearly consistent with the size of ELP-AuNP $(8 \pm 2 \mathrm{~nm})$ — the TEM image (Figure 1)—and that of its small aggregate.

\section{CONCLUSION}

In this study, we have proposed an effective and facile method for preparing self-assembled AuNP arrays guided by thermo-responsive ELP. The ELP-coated AuNPs were successfully prepared by the in situ reduction of $\mathrm{HAuCl}_{4}$ in the presence of lipoic acid-terminated $(\mathrm{VPGVG})_{4}$. The thermo-responsiveness of the as-obtained ELPAuNP was characterized using CD and UV-Vis spectroscopies. The phase transition of the ELP shell proceeded gradually from a random coil to a $\beta$-turn with increasing temperature, similar to the phase transition of the AuNP-free ELP in aqueous solution. This phase transition caused an aggregation of the ELP-AuNP because of hydrophobic interactions and was accompanied by a drastic change in the solution color. Using the hydrophobic character of the ELPAuNP above phase transition temperature, the ELP-AuNPs were selectively aligned onto the patterned hydrophobic PS stripes on a glass plate, as confirmed via AFM and SEM. The method by which the AuNPs were aligned in this study is based on one of the functions of ELP as a 'smart' peptide: the concept is simple and must be applicable to the organization of other metal nanoparticles and organic or bio-nanomaterials.

\section{ACKNOWLEDGEMENTS}

This work was supported in part by a grant-in-aid for scientific research (no 21750130), and by the Strategic Development of Research Infrastructure for Private Universities on 'Creating a Research Center for Advanced Molecular Biochemistry' from the Ministry of Education, Culture, Sports, Science and Technology (MEXT), Japan.
1 Urry, D. W., Luan, C.-H., Harris, C. M. \& Parker, T. Protein-Based Materials (eds McGrath, K. \& Kaplan, D.) (Birkhäuser, Boston, 1997).

2 Manno, M., Emanuele, A., Martorana, V., San Biagio, P. L., Bulone, D., Palma Vittorelli, M. B., MzPherson, D. T., Xu, J., Parker, T. M. \& Urry, D. W. Interaction of processes on different length scales in a bioelestomer capable of performing energy conversion. Biopolymers 59, 51-64 (2001).

3 van Hest, J. C. M. \& Tirrell, D. A. Protein-based materials, toward a new level of structural control. Chem. Commun. 1897-1904 (2001)

4 Foster, J. A., Bruenger, E., Gray, W. R. \& Sandberg, L. B. Isolation and amino acid sequences of tropoelastin peptides. J. Biol. Chem. 248, 2876-2879 (1973).

5 Gray, W. R., Sandberg, L. B. \& Foster, J. A. Molecular model for elastin structure and function. Nature 246, 461-466 (1973)

6 Lee, J., Macosko, C. W. \& Urry, D. W. Elastomeric polypentapeptides cross-linked into matrixes and fibers. Biomacromolecules 2, 170-179 (2001).

7 Lee, J., Macosko, C. W. \& Urry, D. W. Mechanical properties of cross-linked synthetic elastomeric polypentapeptides. Macromolecules 34, 5968-5974 (2001).

8 Nath, N. \& Chilkoti, A. Interfacial phase transition of an environmentally responsive elastin biopolymer adsorbed on functionalized gold nanoparticles studied by colloidal surface plasmon resonance. J. Am. Chem. Soc 123, 8197-8202 (2001).

9 Nagapudi, K., Brinkman, W. T., Leisen, J. E., Huang, L., McMillan, R. A., Apkarian, R. P., Conticello, V. P. \& Chaikof, E. L. Photomediated solid-state cross-linking of an elastinmimetic recombinant protein polymer. Macromolecules 35, 1730-1737 (2002).

10 Wright, E. R., McMillan, R. A., Cooper, A., Apkarian, R. P. \& Conticello, V. P. Thermoplastic elastomer hydrogels via self-assembly of an elastin-mimetic triblock polypeptide. Adv. Funct. Mater. 12, 149-154 (2002)

11 McMillan, R. A., Lee, T. A. T. \& Conticello, V. P. Rapid assembly of synthetic genes encoding protein polymers. Macromolecules 32, 3643-3648 (1999).

12 Dinerman, A. A., Cappello, J., Ghandehari, H. \& Hoag, S. W. Swelling behavior of a genetically engineered silk-elastin like protein polymer hydrogel. Biomaterials 23 4203-4210 (2002)

$13 \mathrm{Li}$, B., Alonso, D. O. V., Bennion, B. J. \& Daggett, V. Hydrophobic hydration is an important source of elasticity in elastin-based biopolymers. J. Am. Chem. Soc. 123, 11991-11998 (2001).

$14 \mathrm{Li}$, B., Alonso, D. O. V. \& Daggett, V. The molecular basis for the inverse temperature transition of elastin. J. Mol. Biol. 305, 581-592 (2001).

15 Takei, Y. G., Aoki, T., Sanui, K., Ogata, N. \& Okano, T. Dymanic contact angle measurement of temperature-responsive surface properties for poly $(\mathrm{N}$-isopropylacrylamide) grafted surfaces. Macromolecules 27, 6163-6166 (1994).

16 Yakushiji, T., Sakai, K., Kikuchi, A., Aoyagi, T., Sakurai, Y. \& Okano, T. Graft architectural effects on thermoresponsive wettability chages of poly $(N$-isopropylacrylamide)-modified surfaces. Langmuir 14, 4657-4662 (1998).

17 Liang, L., Feng, X., Liu, J., Rieke, P. C. \& Fryxell, G. E. Reversible surface properties of glass plate and capillary tube grafted photopolymerization of $\mathrm{N}$-isopropylacrylamide. Macromolecules 31, 7845-7850 (1998).

18 Higashi, N., Takagi, T. \& Koga, T. Layer-bylayer fabrication of well-packed gold nanoparticle assemblies guided by a $\beta$-sheet peptide network. Polym. J. 42, 95-99 (2010)

19 Zehner, R. W., Lopes, W. A., Morkved, T. L., Jaeger, H. \& Sita, L. R. Selective decoration of a phase-separated diblock copolymer with thiol-passivated gold nanocrystals. Langmuir 14, 241-244 (1998)

20 Koga, T., limura, M. \& Higashi, N. Novel peptide-shelled dendrimer with dramatically changeable thermo-responsive character. Macromol Biosci. 12, 1043-1047 (2012).

21 Radha, B. \& Kulkarni, G. U. Dewetting assisted patterning of polystyrene by soft lithography to create nanotrenches for nanomaterial deposition. ACS Appl. Mater Interfaces 1, 257-260 (2009).

22 Brust, M., Walker, M., Bethell, D., Schiffrin, D. J. \& Whyman, R. Synthesis of thiolderivatised gold nanoparticles in a two-phase liquid-liquid system. J. Chem. Soc. Chem. Commun. 801-802 (1994).

23 Elghanian, R., Storhoff, J. J., Mucic, R. C., Letsinger, R. L. \& Mirkin, C. A. Selective colorimetric detection of polynucleotides based on the distance-dependent optical properties of gold nanoparticles. Science 277, 1078-1081 (1997).

24 Lazarides, A. A. \& Schatz, G. C. DNA-linked metal nanosphere materials: structural basis for the optical properties. J. Phys. Chem. B 104, 460-467 (2000). 